\section{Clinical significance of dasatinib-induced pleural effusion in patients with de novo chronic myeloid leukemia}

\author{
Aya Nakaya, Shinya Fujita, \\ Atsushi Satake, Takahisa Nakanishi, \\ Yoshiko Azuma, Yukie Tsubokura, \\ Masaaki Hotta, Hideaki Yoshimura, \\ Kazuyoshi Ishii, Tomoki Ito, \\ Shosaku Nomura
}

First Department of Internal Medicine, Kansai Medical University, Osaka,

Japan

\begin{abstract}
Dasatinib is currently approved for clinical use as a first-line treatment agent for newly diagnosed chronic myeloid leukemia (CML). However, only a few clinical trials have been performed to evaluate dasatinibinduced PE following first-line therapy. We investigated the incidence and clinical features of dasatinib-induced PE following first-line therapy in Japanese CML patients of real world clinical practice settings. Among 22 patients, the median age of PEpositive patients was higher than that of $\mathrm{PE}$ negative patients. Major molecular response was achieved in 75\% of PE-positive patients and $50 \%$ of PE-negative patients. Most patients developed PE more than 1 year after treatment. Appearance of $\mathrm{PE}$ is associated with better clinical response during dasatinib treatment, however it is developed at any time. Elderly and high-risk patients tend to develop PE. The clinical features of dasatinib-induced PE following first-line therapy might be late onset and might not immediately follow the increasing of large granular lymphocyte.
\end{abstract}

\section{Introduction}

Dasatinib, a second-generation tyrosine kinase inhibitor (TKI), is currently approved for clinical use as a first-line treatment agent for newly diagnosed chronic myeloid leukemia (CML). Despite the efficacy and safety of dasatinib, adverse events such as pleural effusion (PE) remain a serious concern. However, a paradoxical relationship between the development of PE and the efficacy of dasatinib has been reported. Patients who developed PE showed a better response rate than did patients without PE. ${ }^{1}$ PE was reported in $15 \%$ to $30 \%$ of patients treated with dasatinib, with a median time to appearance of 5 to 26 weeks. ${ }^{2-6}$ However, with the exception of the DASISION study, ${ }^{7}$ which is the largest phase III trial to date comparing dasatinib to imatinib in patients with newly diagnosed CML, all previous studies of dasatinib-induced PE involved patients receiving dasatinib as second-line therapy. Few studies of dasatinib-induced PE following first-line therapy have been reported. ${ }^{8,9}$ We anticipated that the clinical features of PE following initial use of dasatinib might differ from those associated with second-line use. We herein report the incidence and clinical features of dasatinib-induced $\mathrm{PE}$ following first-line therapy in patients with CML.

\section{Materials and Methods}

In total, 22 consecutive patients diagnosed with chronic-phase CML and treated with dasatinib as initial therapy at Kansai Medical University Hospital from June 2011 to December 2015 were included in this retrospective study. CML diagnosis and efficacy was assessed according to the European Leukemia Net (ELN) 2006 definition. ${ }^{10}$ Molecular response was assessed by quantitative reverse-transcriptase PCR and converted to the International Scale (IS). Peripheral blood smears were examined under light microscopy by two certified hematology technicians. Lymphocytes with three or more large granules per cell were defined as large granular lymphocytes (LGLs), and 200 white blood cells were counted per smear. The LGL count and chest X-rays were monitored every 3 months. When patients developed PE, dasatinib was stopped or the dose was decreased. Patients were also given diuretics to control PE. The dasatinib was then restarted after the PE had diminished. The severity of adverse events was graded using the National Cancer Institute Common Terminology Criteria for Adverse Events, v4.0. ${ }^{11}$ This retrospective analysis was approved by the Ethics Committee of Kansai Medical University.

\section{Results}

The median age of the patients was 55 years (range, 19-75 years), and 95\% were male. We used three major risk stratification scales to predict the outcome: the Sokal score, Hasford score, and EUTOS score. However, most patients were classified as
Correspondence: Aya Nakaya, First Department of Internal Medicine, Kansai Medical University, 2-5-1, Shin-machi, Hirakata, Osaka 573-1010, Japan.

E-mail: +81.72.804.2503.

E-mail: nakaya1016@yahoo.co.jp

Key words: Chronic myeloid leukemia; Dasatinib; large granular lymphocyte; pleural effusion; late-onset; adverse event.

Conflict of interest: the authors declare no conflict of interest.

Received for publication: 1 November 2017. Accepted for publication: 27 June 2018.

This work is licensed under a Creative Commons Attribution-NonCommercial 4.0 International License (CC BY-NC 4.0).

(C) Copyright A. Nakaya et al., 2018

Licensee PAGEPress, Italy

Hematology Reports 2018; 10:7474

doi:10.4081/hr.2018.7474

having low-risk CML according to their Sokal and EUTOS scores (90\% and 92\%, respectively) and low- and intermediaterisk CML according to their Hasford score (low, 55\%; intermediate, $45 \%$ ). The results of a comparison between PE-positive and PE-negative patients are shown in Table 1. The median age of PE-positive patients was higher than that of PE-negative patients (66 vs. 42 years, respectively). Furthermore, all scores were higher in PE-positive patients. Molecular response 4 was achieved in $75 \%$ of PE-positive patients and 50\% of PE-negative patients. The most frequent adverse event was grade 1/2 PE, which was observed in $36 \%$ of patients, followed by grade 1 rash $(9 \%)$, grade 1 QT prolongation (5\%), and grade $1 / 2$ pancytopenia (5\%). Failed treatment occurred in two patients because of poor treatment adherence.

Eight patients developed PE. The characteristics of these patients are shown in Table 2. One patient developed PE 1.4 months after administration of dasatinib, while the others developed PE more than 1 year after treatment; the median time to the development of PE was 17.2 months (range, 1.4-38.7 months). All patients who developed PE achieved major molecular response (MMR). The median cumulative dose of dasatinib was $45.15 \mathrm{~g}$, and all but one patient had an increased LGL count upon development of PE. The median LGL count of patients with PE was $1.980 \times 10^{9} / \mathrm{L}$ (range, $0.647-7.540 \times 10^{9} / \mathrm{L}$ ). The timing of LGL elevation, appearance of PE and the relationship of response were revealed in Figure 1. 


\section{Discussion and Conclusions}

Dasatinib is a second-generation TKI that has been approved for clinical use in first-line therapy. Despite the efficacy and safety of dasatinib, adverse events such as $\mathrm{PE}$ remain a serious concern. Most previous studies that evaluated dasatinib-induced $\mathrm{PE}$ involved patients receiving dasatinib as second-line therapy, and they reported a PE frequency of $15 \%$ to $30 \%$ and median time to appearance of 5 to 26 weeks. ${ }^{2-6}$ The DASISION study, ${ }^{7}$ the largest phase III trial, recently reported the final results of a comparison of dasatinib with imatinib in patients with newly diagnosed CML. ${ }^{12,13}$ In that study, the frequency of PE was $28 \%$, and patients who developed PE tended to be older ( $>65$ years). Patients with PE had a better prognosis, with $96 \%$ achieving a confirmed complete cytogenetic response and $82 \%$ achieving MMR. In another singlearm first-line study in Japan, ${ }^{9} 33 \%$ of patients developed $\mathrm{PE}$, and the MMR rates at 3 months were significantly higher in PEpositive than PE-negative patients ( $54 \%$ vs. $24 \%$, respectively; $\mathrm{P}=0.013$ ).

In this study, the median age of PE-positive patients was higher than that of PEnegative patients (66 vs. 42 years, respectively) (Table 1). As the DASISION study ${ }^{7}$ also reported, age might therefore be a risk factor for PE. Using the risk scales described in the present study, patients with PE had a tendency to be categorized as having higher-risk CML than did PE-negative patients. However, PE-positive patients reached a deeper response than did PE-negative patients. The Sokal score and Hasford score were established before the TKI era. The EUTOS score was constructed using data from patients being treated with imatinib; however, it has not been sufficiently verified. Thus, these scales are necessary to evaluate the predictive potential in the TKI era.

Although the mechanism of PE devel- opment is unknown, most recent reports describe an association between an increased LGL count and PE. ${ }^{14}$ Some studies have further suggested that dasatinibinduced PE may be immune-mediated. ${ }^{5}$ Nagata et al. ${ }^{6}$ reported that elevated LGL counts preceded the development of PE and that patients developed PE approximately 1 month after the appearance of a high LGL count. Mustjoki et al. ${ }^{14}$ also described an elevated LGL count prior to PE, with a median 1.0- to 3.5-month interval from administration of dasatinib to development of PE. In the present study, most patients developed an elevated LGL count at an early stage of dasatinib treatment despite the late onset of PE ( $>1$ year after starting treatment). The increase of LGL is seemed to precede MMR, but the appearance of PE did not necessarily follow increasing of LGL (Figure 1). In the DASISION study, ${ }^{8}$ grade $3 / 4$ pleural effusions was seen after 3 years from the start of treatment. On the other hand, in the CA 180-034 study ${ }^{8}$ including second-line use, grade 3/4 PE was seen during the first year. In our study, most cases developed PE after 1 year from the treatment, suggesting the possibility of delayed onset of PE when dasatinib is used as the first-line. Although increasing of LGL is said to proceed before PE, in this study PE does not develop immediately after the increase in LGL. We assume that the presence of the time lag might be a characteristic of first-line treatment. Months might be required before increased LGL transit from blood to tissue. Thus, the onset of PE might be delayed in the first-line treatment.

Because previous reports have indicated that an elevated LGL count might be associated with PE, we examined the LGL counts

Table 1. Patients' characteristics.

\begin{tabular}{lcc} 
& Patients with PE & Patients without PE \\
No. of patients & 8 & 14 \\
Median age, range (y/0) & $66(54-73)$ & $42(19-75)$ \\
\hline Male sex (\%) & 100 & 95 \\
Sokal score (\%) & & \\
$\quad$ Low & 63 & 95 \\
$\quad$ Intermediate & 25 & 5 \\
$\quad$ High & 12 & 0 \\
\hline Hasford score(\%) & & \\
Low & 25 & 79 \\
Intermediate & 75 & 21 \\
High & 0 & 0 \\
EUTOS score(\%) & & 95 \\
Low & 88 & 5 \\
High & 12 & 50 \\
Best response (\%) & & 7 \\
MR 3.0 & 25 & 29 \\
MR 4.0 & 25 & 14 \\
MR 4.5 & 38 & \\
MR 5.0 & 12 & \\
\hline
\end{tabular}

PE, pleural effusion; MR, molecular response.

Table 2. Characteristics of patients with pleural effusion

\begin{tabular}{|c|c|c|c|c|c|c|}
\hline No. & Sex & Age & Months to PE & LGL (/I) at PE & Cumulative dose of dasatinib (g) & Best response \\
\hline 1 & M & 73 & 12.0 & 1.798 & 42.7 & MR 4.5 \\
\hline 2 & M & 66 & 13.3 & 1.858 & 30.4 & MR 4.0 \\
\hline 3 & $\mathrm{M}$ & 72 & 26.9 & 2.089 & 67.6 & MR 4.5 \\
\hline 4 & M & 54 & 1.4 & 0.647 & 2.1 & MR 3.0 \\
\hline 5 & M & 66 & 18.5 & 2.460 & 55.5 & MR 4.5 \\
\hline 6 & M & 66 & 22.7 & 3.850 & 27.2 & MR 5.0 \\
\hline 7 & M & 71 & 38.7 & 1.872 & 116.2 & MR 3.0 \\
\hline 8 & M & 61 & 15.9 & 7.540 & 47.6 & MR 4.0 \\
\hline
\end{tabular}

M, male; LGL, large granular lymphocytes; PE, pleural effusion; MR, molecular response. 


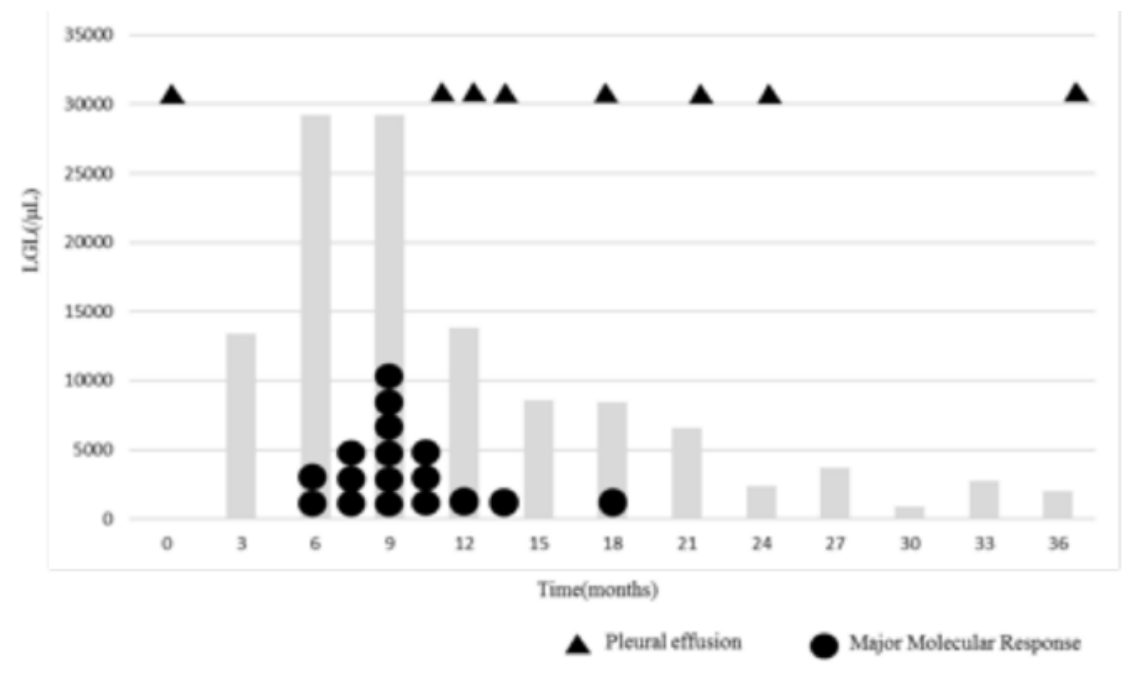

Figure 1. The timing of large granular lymphocytes (LGL) elevation, appearance of pleural effusion (PE) and the relationship of response. MR, molecular response. The increase of LGL is seemed to precede MMR, but the appearance of PE did not necessarily follow increasing of LGL. PE is developed at any time during dasatinib treatment.

in patients who developed PE and found that the median count was $1.980 \times 10^{9} / \mathrm{L}$, which is elevated. In previous studies, the median LGL count ranged from 2.8 to $17.4 \times 10^{9} /$ L. $^{15}$ However, the lower limit associated with the development of PE is unclear. The LGL count might be correlated with the development of PE, although elevated LGL has also been reported in patients without PE. We also evaluated the cumulative dose of dasatinib but found no correlation between the cumulative dose and LGL count (correlation coefficient, 0.0348; $\mathrm{P}=0.935$ ).

Some reports from Asian countries have described differences between Asian and non-Asian patients with CML, with the prevalence of PE slightly higher in patients in Asian countries. In a subset analysis of the DASISION trial with a 2-year followup, PE was reported in $27 \%$ of Japanese patients compared with $13 \%$ of the total patient population. ${ }^{11}$ In the Korean study, PE was observed in $54 \%$ of patients. ${ }^{16}$ Thus, differences might be expected according to the patients' ethnicity and location. Furthermore, Epstein-Barr virus (EBV) infection might be associated with PE because some reports have described EBV-associated PE. ${ }^{17}$

This study had some limitations. It was performed retrospectively, had a small sample size, and involved a single treatment center in Japan. Although our aim was to compare the characteristics of patients treated with dasatinib as a first- versus secondline agent, most patients were conventional- ly treated with nilotinib as a second-line agent in our facility. Therefore, comparison was not possible in these patients.

Although the etiology of PE is unknown, the clinical features of dasatinibinduced PE following first-line therapy might differ from those of second-line therapy. Thus, the clinical significance of PE following first-line therapy warrants further investigation because it appears to represent a long-term adverse event that should be monitored in patients receiving dasatinib.

\section{References}

1. Eskazan AE, Eyice D, Kurt EA, et al. Chronic myeloid leukemia patients who develop grade I/II pleural effusion under second-line dasatinib have better responses and outcomes than patients without pleural effusion. Leuk Res 2014;38:781-7.

2. Cortes J, Rousselot P, Kim DW, et al. Dasatinib induces complete hematologic and cytogenetic responses in patients with imatinib-resistant or -intolerant chronic myeloid leukemia in blast crisis. Blood 2007;109:3207-13.

3. Guilhot F, Apperley J, Kim DW, et al. Dasatinib induces significant hematologic and cytogenetic responses in patients with imatinib-resistant or intolerant chronic myeloid leukemia in accelerated phase. Blood 2007;109: 4143-50.

4. Hochhaus A, Baccarani M, Deininger $\mathrm{M}$, et al. Dasatinib induces durable cytogenetic responses in patients with chronic myelogenous leukemia in chronic phase with resistance or intolerance to imatinib. Leukemia 2008;22: 1200-6.

5. Quintás-Cardama A, Kantarjian H, O'brien S, et al. Pleural effusion in patients with chronic myelogenous leukemia treated with dasatinib after imatinib failure. J Clin Oncol 2007;25: 3908-14.

6. Nagata Y, Ohashi K, Fukuda S, et al. Clinical features of dasatinib-induced large granular lymphocytosis and pleural effusion. Int J Hematol 2010;91: 799-807.

7. Kantarjian H, Shah NP, Hochhaus A, et al. Dasatinib versus imatinib in newly diagnosed chronic-phase chronic myeloid leukemia. N Engl J Med 2010;362:2260-70.

8. Cortes JE, Jimenez CA, Mauro MJ, et al. Pleural effusion in dasatinib-treated patients with chronic myeloid leukemia in chronic phase: identification and management. Clin Lymphoma Myeloma Leuk 2017;17:78-82.

9. Hagihara M, Iriyama N, Yoshida C, et al. Association of pleural effusion with an early molecular response in patients with newly diagnosed chronic-phase chronic myeloid leukemia receiving dasatinib: Results of a D-First study. Oncol Rep 2016;36:2976-82.

10. Baccarani M, Saglio G, Goldman J, et al. Evolving concepts in the management of chronic myeloid leukemia: recommendations from an expert panel on behalf of the European Leukemia Net. Blood 2006;108:1809-20.

11. National Cancer Institute. Common terminology criteria for adverse events (CTCAE). Last Updated: 03/01/18. Available from: http://ctep.cancer.gov/protocolDevelop ment/electronic_applications/ctc.htm\#c tc_40 Accessed: September 1, 2017.

12. Cortes JE, Saglio G, Kantarjian HM, et al. Final 5-year study results of DASISION: the dasatinib versus imatinib study in treatment-naïve chronic myeloid leukemia patients trial. J Clin Oncol 2016;34:2333-40.

13. Fujisawa S, Nakamae H, Ogura M, et al. Efficacy and safety of dasatinib versus imatinib in Japanese patients with newly diagnosed chronic-phase chronic myeloid leukemia (CML-CP): Subset analysis of the DASISION trial with 2year follow-up. Int $\mathrm{J}$ Hematol 2014;99:141-53.

14. Mustjoki S, Ekblom M, Arstila TP, et al. Clonal expansion of T/NK-cells during tyrosine kinase inhibitor dasatinib ther- 
apy. Leukemia 2009;23:1398-405.

15. Kim DH, Kamel-Reid S, Chang H, et al. Natural killer or natural killer/T cell lineage large granular lymphocytosis associated with dasatinib therapy for Philadelphia chromosome positive leukemia. Haematologica 2009;94:1359.

16. Kim D, Goh HG, Kim SH, et al. Longterm pattern of pleural effusion from chronic myeloid leukemia patients in second-line dasatinib therapy. Int $\mathrm{J}$
Hematol 2011;94:361-71.

17. Takei H, Mody D. Epstein-Barr viruspositive pleural effusion: clinical features, cytomorphologic characteristics, and flow cytometric immunophenotyping. Am J Clin Pathol 2014;142:788-94. 